\title{
Celiac Plexus Neurolysis for the Treatment of Patients with Terminal Cancer at a Tertiary University Hospital in Korea
}

\section{Gyeong-Jo Byeon, M.D., Ph.D., Ju Yeon Park, M.D., Yun-Mi Choi, M.D., Hyun-Su Ri, M.D., Ph.D., Ji-Uk Yoon, M.D., Ph.D. and Eun-Ji Choi, M.D.}

Department of Anesthesia and Pain Medicine, Pusan National University Yangsan Hospital, Pusan National University School of Medicine, Research Institute for Convergence of Biomedical Science and Technology, Pusan National University Yangsan Hospital, Yangsan, Korea

Purpose: The aim of this study was to investigate celiac plexus neurolysis (CPN) for the treatment of cancerous upper abdominal pain in a tertiary university hospital in Korea. Methods: At the tertiary university hospital in Korea, electronic medical records of cancer patients who underwent CPN and died in the hospital from November 2009 to June 2018 were retrospectively analyzed. Results: The total number of subjects was 51 . The 17 patients were from the Department of Gastroenterology (33.0\%), followed by 11 patients from the Department of Hemato-oncology (21.6\%), 11 patients from the Department of Anesthesia and Pain Medicine (21.6\%), 9 patients from the Department of General Surgery (17.6\%). The diagnosis was pancreatic cancer in 15 patients (29.4\%), stomach cancer in 8 patients (15.7\%), hepatobiliary cancer in 20 patients (39.2\%), colon cancer in 1 patient (2.0\%), esophageal cancer in 2 patient (3.9\%) and intra-abdominal metastasis in 5 patients (9.8\%). The mean survival time after the surgery was $66.4 \pm 55.0$ days. The pain intensity before and 1 week after the procedure significantly decreased, but the amounts of opioids consumed before and 1 week after the procedure were not statistically significant. Side effects occurred after the procedure including temporary localized pain in 24 patients (47.0\%), hypotension in $12(23.5 \%)$, and diarrhea in $6(11.8 \%)$. Conclusion: $\mathrm{CPN}$ is an effective and safe procedure for reducing upper abdominal pain caused by cancer, and it is necessary to perform CPN within the appropriate time by establishing a system of interdepartmental $\mathrm{CO}^{-}$ operation.

Key Words: Abdominal pain, Analgesia, Celiac plexus, Cancer pain, Pain management
Received April 26, 2019

Revised August 28, 2019

Accepted October 24, 2019

\section{Correspondence to}

Eun-Ji Choi

Department of Anesthesia and Pain Medicine, Pusan National University School of Medicine, Geumo-ro 20, Mulgeumeup, Yangsan, Gyeongnam 50612, Korea

Tel: +82-55-360-1622

Fax: +82-55-360-2149

E-mail: eunjichoi81@gmail.com

This work was supported by clinical research grant from Pusan National University Yangsan Hospital in 2018.

\section{INTRODUCTION}

Pancreatic cancer and other malignant tumors of the upper abdominal organs often cause difficult-to-control abdominal pain (1). For pain control, doctors generally follow the World Health Organization recommendations and rely heavily on combinations of NSAIDs, acetaminophen, and narcotic anal- gesics. However, this guideline alone cannot effectively control pain, as many patients complain of inadequate pain control (2-4). In addition, some patients can have severe side effects even with a small amount of opioid analgesics, continuing which can increase the side effects (5). Thus, pain control through invasive procedures is required (6).

Celiac plexus neurolysis (CPN) is a procedure performed 
to reduce cancer-related upper abdominal pain (7). CPN is a useful and safe method, which is being widely used now. However, conflicting results on its effects have been reported by several studies (8-10), and data are insufficient on CPN treatment in tertiary university hospitals in Korea.

This study aimed at analyzing the course of CPN procedure for cancer-related upper abdominal pain and the results after the procedure at a tertiary university hospital.

\section{METHODS}

After receiving approval from the Institutional Review Board (Approval No. 05-2018-146), electronic medical records were used to conduct a retrospective study on patients who underwent CPN for pain in the peritoneal cavity from November 2009 to June 2018. Patients with abdominal pain due to cancer had been referred from various departments to the pain clinic to undergo CPN, as opioid analgesics could not control their abdominal pain or the opioid dose could not be increased because of the side effects. The referred patients had terminal cancer and needed palliative treatment. In the patients referred for abdominal pain, CPN was not performed because of a risk of bleeding during the procedure in those who were undergoing anticoagulant therapy, had a platelet count $\leq 100,000$, or had prolonged PT or PPT. Among the 101 patients who underwent CPN, three who had no post-procedural records and 47 whose survival period was unknown because of transfer were excluded, resulting in a total of 51 patients in the present study.
Before the procedure, each patient provided informed consent and received $500 \mathrm{~mL} \sim 1,000 \mathrm{~mL}$ of balanced salt solution for intravenous fluid hydration. Patients were monitored for blood pressure, electric activity of the heart using electrocardiography, and oxygen saturation. An intradiscal approach was used in CPN.

A pillow was placed on the patient abdomen in the prone position to reduce lordosis. The location of the T12-L1 level was confirmed by fluoroscopy. The location of the superior articular process of the 1st lumbar vertebra was confirmed in the oblique view within 35 40 degrees of C-arm fluoroscopy. After local infiltration with $1 \%$ lidocaine under sterile conditions, a 22-G, 15-cm sized nerve blockade needle (Hakko Co. Ltd, Nagano, Japan) was inserted in the superior articular process of the 1 st lumbar vertebra, and the $\mathrm{C}$-arm lateral view was confirmed when it reached the superior articular process. The needle was advanced to the back of the disc and connected to the syringe containing normal saline and advanced slowly when the needle reached the anterior border of the vertebral body through the disc. The needle tip position was confirmed with $5 \mathrm{~mL}$ of contrast media (Pamiray 300; Dongkook Pharmaceutical, Seoul, Korea), and $20 \mathrm{~mL}$ of $1 \%$ lidocaine was injected (Figure 1). After lidocaine injection, we observed whether side effects such as intravascular injection occurred. If no neurological adverse events were observed and pain decreased, $20 \mathrm{~mL}$ of $99 \%$ ethyl alcohol was slowly injected.

After the procedure, patients took absolute rest and were continuously monitored for blood pressure, electrical activity of the heart using electrocardiography, and oxygen saturation.
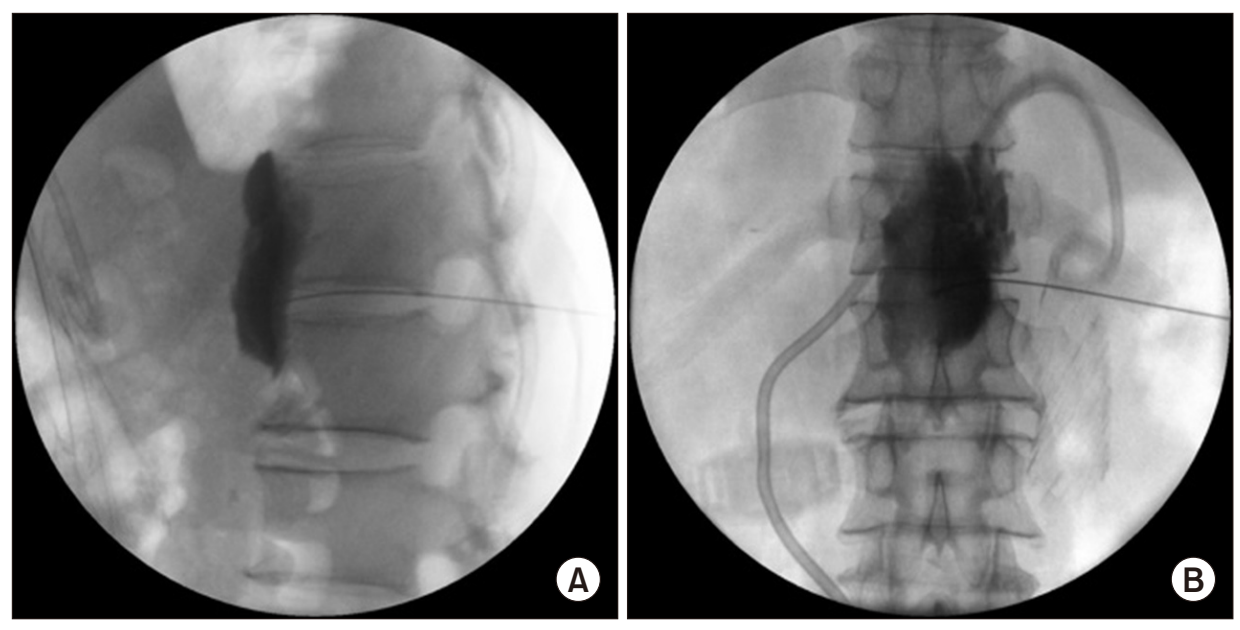

Figure 1. Fluoroscopic images of contrast spread after intradiscal celiac plexus neurolysis. (A) Fluoroscopic lateral view of a single needle placement of the celiac plexus with the contrast confirming the correct position anterior to the vertebral body. (B) Fluoroscopic anteroposterior view of contrast spread. 
When the patients were hemodynamically stable, they were transferred to a ward.

To increase the accuracy of data collection, two investigators reviewed patient charts and collected data, and a third investigator analyzed the data. The same investigators conducted the study.

Data on the patient's sex, age, height, bodyweight, admission department, type of cancer, and survival time after the procedure were collected. Pain before and 1 week after the procedure was assessed using the 11-point numerical rating scale (NRS-11) score. Opioid consumption was determined by computing the morphine equivalent daily dose (MEDD). To standardize the opioid dosage, opioid consumption over a 24hour period was converted to oral morphine equivalent $(11,12)$. The dosage of oral morphine before and 1 week after the procedure was calculated. Records of complications from the procedure, such as transient local pain, hypotension, diarrhea, other paraplegia, renal injury, and discitis, were also collected from immediately after the procedure to 1 week after the procedure. Severities of hypotension and diarrhea were classified based on the Common Terminology Criteria for Adverse Events (CTCAE) version 4.03. Hypotension was defined as blood pressure below 90/60 mmHg and CTCAE version 4.03 grade $\geq 2$. Diarrhea was defined as four or more loose stools a day with CTCAE version 4.03 grade $\geq 2$ (13).

All continuous variable data were reported as mean \pm standard deviation (SD). The number of patients, patient's admission department, types of cancer, and adverse events were presented as numbers and proportions. Data distribution was evaluated using the Kolmogorov-Smirnov test. Continuous variables were analyzed using the paired t-test. All statistical analyses were performed using the Statistical Package for the Social Sciences (SPSS) software version 18.0 for Windows. P values less than 0.05 were considered statistically significant.

\section{RESULTS}

A total of 51 patients who underwent CPN in this hospital and died because of cancer were enrolled in the retrospective study. There were 30 men and 21 women, and the mean age was $57.9 \pm 11.8$ years. The average height of the patients was $162.6 \pm 7.8 \mathrm{~cm}$ and the mean weight was $54.1 \pm 10.2 \mathrm{~kg}$. Sev- enteen patients were from the Department of Gastroenterology (33.0\%), followed by 11 patients from the Department of Hemato-oncology (21.6\%), 11 patients from the Department of Anesthesia and Pain Medicine (21.6\%), 9 patients from the Department of General Surgery (17.6\%), 1 patient from the Department of Obstetrics and Gynecology (2.0\%), 1 patient from the Department of Pulmonology (2.0\%), and 1 patient from the Department of Family Medicine (2.0\%). The diagnosis was pancreatic cancer in 15 patients (29.4\%), stomach cancer in 8 patients (15.7\%), hepatobiliary cancer in $20 \mathrm{pa}^{-}$ tients (39.2\%), colon cancer in 1 patient (2.0\%), esophageal cancer in 2 patient $(3.9 \%)$ and intra-abdominal metastasis in 5 patients (9.8\%). Two of the five patients with an intraabdominal metastasis had lung cancer, and the remaining three had ovarian, cervical, and urethral cancers, respectively. The mean survival time after procedure was $66.4 \pm 55.0$ days (Table 1).

The NRS pain scores before and 1 week after the procedure were $5.8 \pm 1.0$ and $3.6 \pm 1.4$, respectively. The NRS score was

Table 1. Patient Characteristics.

\begin{tabular}{|c|c|}
\hline Characteristics & $\mathrm{n}(\%)$ \\
\hline \multicolumn{2}{|l|}{ Sex } \\
\hline Men & 30 \\
\hline Women & 21 \\
\hline Age $(y r$, mean $\pm S D)$ & $57.9 \pm 11.8$ \\
\hline Height (cm, mean $\pm S D$ ) & $162.6 \pm 7.8$ \\
\hline Weight (kg, mean $\pm S D$ ) & $54.1 \pm 10.2$ \\
\hline \multicolumn{2}{|l|}{ Admission department } \\
\hline Gastroenterology & $17(33.0)$ \\
\hline Hemato-oncology & $11(21.6)$ \\
\hline Anesthesia and pain Medicine & $11(21.6)$ \\
\hline General surgery & $9(17.6)$ \\
\hline Obstetrics and gynecology & $1(2.0)$ \\
\hline Pulmonology & $1(2.0)$ \\
\hline Family medicine & $1(2.0)$ \\
\hline \multicolumn{2}{|l|}{ Cancer type } \\
\hline Pancreatic cancer & $15(29.4)$ \\
\hline Stomach cancer & $8(15.7)$ \\
\hline Hepatobiliary cancer & $20(39.2)$ \\
\hline Colon cancer & $1(2.0)$ \\
\hline Esophageal cancer & $2(3.9)$ \\
\hline Intra-abdominal metastasis & $5(9.8)$ \\
\hline Survival time after procedure (day, mean \pm SD) & $66.4 \pm 55.0$ \\
\hline
\end{tabular}

All measured values are presented as mean \pm standard deviation or number of patients. 
significantly lower 1 week after the procedure than before $(\mathrm{P}<0.05)$. The NRS score decreased in 44 patients $(86.3 \%)$ and remained unchanged in seven patients (13.7\%), with none of the patients showing an increase in the NRS score. MEDD was $331.6 \pm 372.1 \mathrm{mg}$ before the procedure and $275.2 \pm 285.5$ $\mathrm{mg}$ one week after the procedure, but the difference was not statistically significant. Opioid consumptions had no change before and 1 week after the procedure in 10 of the 51 patients and increased in 9 patients. Although not statistically significant, opioid consumption decreased in 32 patients (Table 2).

Among the complications of the procedure, transient local pain occurred most frequently, in 24 (47\%) of the 51 patients. Transient local pain persisted for an average of 36 hours, and the NRS score was $7.3 \pm 1.2$. Hypotension occurred in 12 $(23.5 \%)$ and diarrhea in $6(11.8 \%)$ of the 51 patients. No other serious complications, such as paraplegia, renal injury, or discitis, were observed (Table 3).

\section{DISCUSSION}

Abdominal pain caused by primary cancer or metastatic diseases is the cause of severe pain in patients. Such pain may not be sufficiently ameliorated by conventional analgesics (24). $\mathrm{CPN}$ is commonly performed in patients with uncontrolled abdominal pain caused by pancreatic, gastric, esophageal, or biliary malignancy, as well as metastatic liver cancer and malignancy associated with retroperitoneal lymph node metastasis (7,14-16). The purpose of our study was to investigate CPN for the treatment of cancerous abdominal pain. This study provided several key results.

First of all, most patients had been referred to the pain clinics from other departments. The highest number of patients were referred from the Department of Gastroenterology, and many patients were referred from the Department of Hemato- oncology, Department of Anesthesia and Pain Medicine, and Department of General Surgery. This shows that various departments are currently treating cancer patients. The most common condition was cancer of the upper abdominal organs, followed by pancreatic cancer and abdominal metastasis. Although CPN has been researched extensively for use in pancreatic cancer patients $(10,17)$, our study revealed that it has been most frequently used for those with upper abdominal organ cancer. This shows that CPN has wide applications for the treatment of abdominal cancer pain as well as pancreatic cancer pain.

The mean survival time of patients after getting referred to the pain clinic and undergoing the procedure for abdominal pain was $66.4 \pm 55.0$ days. After the procedure, the patients died within 8 218 days. This large variation in survival time suggests that a long time was taken to decide on CPN for some patients. CPN is a common procedure that is effective for cancerous upper abdominal pain that cannot be controlled with opioid analgesics or in patients who cannot withstand an increased dosage because of adverse reactions, but CPN referrals are relatively few in Korea. Few CPN referrals in Korea seem to be because of the gap between the patient's admission department and the operation department. In addition, CPN seems to be contraindicated in many referred patients, as they have a bleeding tendency or are in a weak state. CPN

Table 3. Incidence of Adverse Effects.

\begin{tabular}{lc}
\hline \multicolumn{1}{c}{ Characteristics } & Number of patients (\%) \\
\hline Transient local pain & $24(47.0)$ \\
Hypotension & $12(23.5)$ \\
Diarrhea & $6(11.8)$ \\
Paraplegia & $0(0.0)$ \\
Renal injury & $0(0.0)$ \\
Discitis & $0(0.0)$ \\
\hline
\end{tabular}

Values are the number of patients (\%).

Table 2. Morphine Equivalent Daily Dose and Pain Scale Before CPN and After CPN.

\begin{tabular}{|c|c|c|c|}
\hline & Before CPN & $\begin{array}{c}\text { After CPN } \\
\text { (1 week) }\end{array}$ & P-value \\
\hline Morphine equivalent daily dose (Oral Morphine, mg) & $331.6 \pm 372.1$ & $275.2 \pm 285.5$ & 0.392 \\
\hline Pain scale (NRS) & $5.8 \pm 1.0$ & $3.6 \pm 1.4$ & $<0.001$ \\
\hline
\end{tabular}

CPN: celiac plexus neurolysis, NRS: numeric rating scale.

All measured values are presented as mean \pm standard deviation. 
is known to provide pain relief for an average of 3 months or more, and for some patients, the pain-relief effect persists till death (14). Therefore, it is necessary to share patient information and present the patient's condition through interdepartmental cooperation before they worsen. The procedure should be performed as early as possible, by multidisciplinary cooperation, for patients with cancer pain.

The NRS score was significantly reduced after 1 week compared to that before the procedure, but there were no statistical differences in opioid consumption. Among 51 patients, opioid consumption decreased in 32. Although not statistically significant, CPN effectively reduced the subjective pain score and opioid consumption in more than half the patients. However, opioid consumption did not significantly decrease following CPN despite a decrease in the NRS score, suggesting that in many patients, the opioid dose could not be sufficiently increased due to the adverse effects of opioid analgesics.

Finally, complications from the procedure occurred as transient local pain after the procedure most frequently, followed by hypotension and diarrhea. Side effects of CPN were reported as local pain (96.0\%), diarrhea (44.0\%) and hypotension (38.0\%) (14). Our study reported much fewer hypotensive cases than previous studies, which is thought to be the result of sufficient hydration before the procedure to prevent hypotension that could cause extensive sympathetic block. Diarrhea was not a concern because most patients with cancerous pain have constipation due to opioid analgesics. There were no other serious complications, such as paraplegia, renal injury, or discitis. There are several approaches for CPN. In this study, CPN was performed using an intradiscal approach with a single needle advanced to the celiac plexus through the T12-L1 intervertebral disc. This approach is used when the peritoneal malignancies have metastasized and the anatomical structure has changed, limiting access to the celiac plexus. Using this approach could prevent organ damage, such as renal injury. However, this approach has been reported to cause diskitis, disc herniation, and spinal cord injury because of the inevitable damage to the disc. However, in this study, intervertebral disc puncture did not cause any complications (16,18-20).

Our study has some limitations. First, this study was a single-center retrospective study, and it is necessary to conduct multi-center prospective studies. Second, the quality of life influenced by pain score reduction was not assessed, although cancerous pain greatly affects the quality of life, and future studies are required. This paper reports the results of a retrospective analysis of medical records; long-term effects up to 3 postoperative months could not be assessed as no data were available for the accurate time points. Finally, this study only included those patients who died at our institution, since the collection of information regarding the duration of postprocedural survival was difficult. The overall survival duration after CPN needs to be calculated by including data from other institutions. With an exception of three patients who lacked post-procedural medical records, most of the 50 patients excluded from the analysis were those who decided to stop aggressive cancer care. Many of these patients were transferred to other institutions for palliative care, so a shorter life expectancy is speculated in them compared to those who died at our institution. Thus, the post-procedural survival period analyzed in this study could have been overestimated.

In conclusion, CPN is an effective and safe procedure reducing upper abdominal cancerous pain, and it is necessary to establish an interdepartmental cooperation system to treat patients with upper abdominal cancerous pain and to perform $\mathrm{CPN}$ in a timely manner.

\section{REFERENCES}

1. Bowles MJ, Benjamin IS. ABC of the upper gastrointestinal tract: Cancer of the stomach and pancreas. BMJ 2001;323:1413-6.

2. Azevedo Sao Leao Ferreira K, Kimura M, Jacobsen Teixeira M. The WHO analgesic ladder for cancer pain control, twenty years of use. How much pain relief does one get from using it? Support Care Cancer 2006;14:1086-93.

3. Portenoy RK. Treatment of cancer pain. Lancet 2011;377:2236-47.

4. Seo MS, Shim JY. Breakthrough cancer pain. Korean J Hosp Palliat Care 2015;18: 1-8.

5. Benyamin R, Trescot AM, Datta S, Buenaventura R, Adlaka R, Sehgal N, et al. Opioid complications and side effects. Pain Physician 2008;11 (2 
Suppl):S105-20.

6. de Oliveira R, dos Reis MP, Prado WA. The effects of early or late neurolytic sympathetic plexus block on the management of abdominal or pelvic cancer pain. Pain 2004;110:400-8.

7. Nagels W, Pease N, Bekkering G, Cools F, Dobbels P. Celiac plexus neurolysis for abdominal cancer pain: a systematic review. Pain Med 2013;14:1140-63.

8. Wong GY, Schroeder DR, Carns PE, Wilson JL, Martin DP, Kinney MO, et al. Effect of neurolytic celiac plexus block on pain relief, quality of life, and survival in patients with unresectable pancreatic cancer: a randomized controlled trial. JAMA 2004;291:1092-9.

9. Fujii-Lau LL, Bamlet WR, Eldrige JS, Chari ST, Gleeson FC, Abu Dayyeh BK, et al. Impact of celiac neurolysis on survival in patients with pancreatic cancer. Gastrointest Endosc 2015;82:46,56.e2.

10. Oh TK, Lee WJ, Woo SM, Kim NW, Yim J, Kim DH. Impact of celiac plexus neurolysis on survival in patients with unresectable pancreatic cancer: A retrospective, propensity score matching analysis. Pain Physician 2017;20:E357-65.

11. Berdine HJ, Nesbit SA. Equianalgesic dosing of opioids. J Pain Palliat Care Pharmacother 2006;20:79-84.

12. Shaheen PE, Walsh D, Lasheen W, Davis MP, Lagman RL. Opioid equianalgesic tables: are they all equally dangerous? J Pain Symptom Manage 2009;38:409-17.

13. National Cancer Institute (U.S.). Common terminology criteria for adverse events (CTCAE) v 4.03. [Bethesda, Md.] :U.S. Department of Health and Human Services;2010.

14. Eisenberg E, Carr DB, Chalmers TC. Neurolytic celiac plexus block for treatment of cancer pain: a meta-analysis. Anesth Analg 1995;80:290-5.

15. Koyyalagunta D, Engle MP, Yu J, Feng L, Novy DM. The effectiveness of alcohol versus phenol based splanchnic nerve neurolysis for the treatment of intra-abdominal cancer pain. Pain Physician 2016;19:281-92.

16. Wang PJ, Shang MY, Qian Z, Shao CW, Wang JH, Zhao XH. CT-guided percutaneous neurolytic celiac plexus block technique. Abdom Imaging 2006;31:710-8.

17. Dobosz L, Stefaniak T, Dobrzycka M, Wieczorek J, Franczak P, Ptaszynska D, et al. Invasive treatment of pain associated with pancreatic cancer on different levels of WHO analgesic ladder. BMC Surg 2016;16:20.

18. Kong YG, Shin JW, Leem JG, Suh JH. Computed Tomography (CT) simulated fluoroscopy-guided transdiscal approach in transcrural celiac plexus block. Korean J Pain 2013;26:396-400.

19. Ina H, Kitoh T, Kobayashi M, Imai S, Ofusa Y, Goto H. New technique for the neurolytic celiac plexus block: the transintervertebral disc approach. Anesthesiology 1996;85:212-7.

20. ErdineS. Celiac ganglion block. Agri 2005;17:14-22. 